\title{
EXTRINSIC RIGIDITY THEOREMS FOR COMPACT SUBMANIFOLDS OF THE SPHERE
}

\author{
ROBERT C. REILLY
}

\section{Introduction}

In this paper we consider immersions $X: M \rightarrow S^{m}$ of a compact oriented Riemannian $n$-manifold $M$ into the standard unit $m$-sphere $S^{m}$, and wish to find conditions on $X$ which imply that it is a standard isometric immersion of a constant curvature $n$-sphere into $S^{m}$, i.e., to find extrinsic rigidity theorems. Our principal tools are certain integral formulas.

In $\S 1$ we briefly discuss the problem of finding interesting integral formulas. As an example we derive a simple integral formula relating the scalar curvature to infinitesmal conformal transformations.

In $\S 2$ we derive some integral formulas for compact hypersurfaces of $S^{n+1}$ (Theorem A) by means of a variant of Newton's formula, and use these integral formulas to prove our first rigidity theorem (Corollary A).

In $\S 3$ we generalize the first two formulas of Theorem $A$ to the case of arbitrary codimension (Theorem B) and then derive an improvement of a rigidity theorem, originally due to De Giorgi and Simons, for compact minimal submanifolds of the sphere whose normal spaces are close enough to each other (Theorem C).

In the appendix we prove a weaker form of Theorem $\mathrm{C}$ using the theory of elliptic partial differential equations (Theorem D).

Notation and conventions. The inner product and norm in the Euclidean space $E^{m+1}$ of dimension $m+1$ are denoted by (, ) and | , respectively. All manifolds are assumed to be connected, and all immersions to be isometric. We denote the directional derivative of an $E^{m+1}$-valued function $f$ along a vector $v$ by $\nabla_{v} f$, which means componentwise differentiation. If $e_{1}, \cdots, e_{n}$ form a frame field for $M$ we shall at times use the notation $f_{, j}$ for $\nabla_{e_{j}} f$, particularly if $f$ is a component of a tensor. In all sections but the appendix we follow the index convention $1 \leq i, j, k, l \leq n$.

The author wishes to thank his advisor, Professor Shiing-Shen Chern, for many helpful suggestions and kind encouragement during the preparation of his dissertation, of which this paper forms a part. He also wishes to thank Professors Robert Gardner and David Gilbarg for several informative conversations.

Received October 4, 1969. The research for this paper was partially supported by an NSF Fellowship. 


\section{General remarks about integral formulas}

Most of the interesting and useful integral formulas in Riemannian geometry follow from Stokes' theorem. That is, one considers a smooth vector field $W$ on a compact oriented Riemannian manifold $M$ with volume element $d V$; the integral formula is $\int_{M} \operatorname{div}(W) d V=0$. The interesting integral formulas therefore correspond to the vector fields on $M$ having interesting divergences. Unfortunately there are no general methods for finding such vector fields, for there exist so many dull vector fields that one must be fairly lucky to find a good example. Because of this it is reasonable to restrict one's attention to some special class of vector fields which (hopefully) contains a higher percentage of good examples.

If we consider a few of the well known integral formulas, e.g., in [1], [7], or [8], we notice that the vector fields considered are of the form $T\left(W^{\prime}\right)$, where $T$ is a symmetric tensor field of type $(1,1)$ (i.e., $T$ is a smooth field of selfadjoint linear operators in the tangent spaces of $M$ ) whose definition may be complicated but such that $\operatorname{div}(T)=0$, and $W^{\prime}$ is a fairly uncomplicated smooth vector field on $M$. We see that the restriction $\operatorname{div}(T)=0$ is reasonable. For let $e_{1}, \cdots, e_{n}$ be an orthonormal frame field which is geodesic at a point $q \epsilon$ $M$. Let $\left(t_{i_{j}}\right)$ be the matrix of $T$ relative to the basis $e_{1}, \cdots, e_{n}$ and let $W_{j}^{\prime}=$ $\left(W^{\prime}, e_{j}\right)$. Then

$$
\begin{aligned}
\operatorname{div}(W)(q) & =\left(\operatorname{div}(T), W^{\prime}\right)+\sum_{i, j} t_{i j} W_{i, j}^{\prime} \\
& =\frac{1}{2} \sum_{i, j} t_{i j}\left(W_{i, j}^{\prime}+W_{j, i}^{\prime}\right),(\text { everything evaluated at } q),
\end{aligned}
$$

since $T$ is symmetric and $\operatorname{div}(T)=0$.

Thus our program is this: a) find a symmetric tensor $T$ of type $(1,1)$ on $M$ such that $\operatorname{div}(T)=0$; b) find a vector field $W^{\prime}$ having interesting properties when combined with $T$.

Example. In the situation considered above, let $\left(R_{i j}\right)$ be the matrix of the Ricci tensor relative to the frame field $e_{1} \cdots e_{n}$ and let $R=\sum_{j} R_{j i}$ be the scalar curvature function. It is well known (cf. [3]) that $\sum_{j} R_{i j, j}=\frac{1}{2} R_{, i}$ at $q$. Thus if $T$ is the symmetric tensor field on $M$ with matrix $t_{i j}=\frac{1}{2} R \delta_{i j}-R_{i j}$ relative to the given frame field, then $\operatorname{div}(T)=0$. Now suppose that $W^{\prime}$ is an infinitesimal conformal transformation on $M$ (cf. [3]); that is, the elements of the one parameter group generated by $W^{\prime}$ are conformal transformations of $M$. Then $W_{i, j}^{\prime}+W_{j, i}^{\prime}=(2 / n) \operatorname{div}\left(W^{\prime}\right) \delta_{i j}$. Thus

$$
\begin{aligned}
n \cdot \operatorname{div}\left(T\left(W^{\prime}\right)\right) & =\sum_{i, j}\left(\frac{1}{2} R \delta_{i j}-R_{i j}\right) \frac{1}{2}\left(W_{i, j}^{\prime}+W_{j, i}^{\prime}\right) \\
& =((n-2) / 2) R \operatorname{div}\left(W^{\prime}\right) .
\end{aligned}
$$


Since $\int_{M} \operatorname{div}\left(T\left(W^{\prime}\right)\right) d V=0$ we have:

Proposition A. If $M$ is a compact oriented Riemannian manifold of dimension $n>2$ with volume element $d V$ and scalar curvature $R$, and if $W$ is any infinitesimal conformal transformation on $M$, then $\int_{M} R \cdot \operatorname{div}(W) d V=0$.

\section{Integral formulas for hypersurfaces of $\boldsymbol{S}^{n+1}$}

Theorem A. Let $X: M \rightarrow S^{n+1}$ be an immersion of the compact orientable $n$-dimensional manifold $M$ as a hypersurface in the unit $(n+1)$-sphere $S^{n+1}$ $\subset E^{n+2}$. Let $N$ be the unit normal vector field of $M$, and $\sigma_{1}, \sigma_{2}, \cdots \sigma_{n}$ be the mean curvature functions on $M$. Let $A$ be any fixed element of $S^{n+1}$, and set $U=(N, A)$ and $V=(X, A)$. Then for $r=0,1, \cdots,(n-1)$ we have:

$$
\int_{M}\left(U \sigma_{r+1}-V \sigma_{r}\right) d V=0 .
$$

$\left(\right.$ We set $\left.\sigma_{0}=1\right)$.

The proof of the theorem is based on the following lemmas.

Lemma A. Suppose that $\left(b_{i j}\right)$ is an $n \times n$ symmetric matrix of differentiable real-valued functions on an open set in Euclidean $m$-space. Let $S_{j}$ be the $j$-th elementary symmetric function of the eigenvalues of $\left(b_{i j}\right)$, and $b_{k l}^{r}$ be the $(k, l)$-th element of the $r$-th power of the matrix $\left(b_{i j}\right)$. Then:

a)

$$
r \nabla_{x} S_{r+1}=\sum_{i, j} b_{i j} \nabla_{x}\left(S_{r} \delta_{i j}-S_{r-1} b_{i j}^{1}+\cdots+(-1)^{r} b_{i j}^{r}\right)
$$

for any vector $x$ in the domain of $\left(b_{i j}\right)$;

b) $(r+1) S_{r+1}=\sum_{j}\left(S_{r} b_{j j}^{1}-S_{r-1} b_{j j}^{2}+S_{r-2} b_{j j}^{3} \cdots+(-1)^{r} b_{j j}^{r+1}\right)$.

Lemma B. Let $\left(b_{i j}\right)$ be the matrix, relative to a local orthonormal frame field $e_{1}, \cdots, e_{n}$ which is geodesic at $q \in M$, of the second fundamental form of a hypersurface $M$ in a Riemannian manifold $M^{\prime}$ of constant sectional curvature. Let $S_{r}$ be the $r$-th elementary symmetric function of the principal curvatures of $M$ in $M^{\prime}$, and let $b_{k l}^{r}$ denote the $(k, l)$-element of the $r$-th power of the matrix $\left(b_{i j}\right)$. Then for each $i=1,2, \cdots n$ we have at $q$ :

$$
\nabla_{e_{i}} S_{r+1}=\sum_{j} \nabla_{e_{j}}\left(S_{r} b_{i j}^{1}-S_{r-1} b_{i j}^{2}+\cdots+(-1)^{r} b_{i j}^{r+1}\right)
$$

Remark. These lemmas are correct for all integers $r$ if we define $S_{r}=0$ for $r>n$.

Proof of Lemma A. a) Let us consider the quantity 


$$
\begin{aligned}
Q_{r} & =\sum_{i, j} b_{i j} \nabla_{x}\left(S_{r} \delta_{i j}-S_{r-1} b_{i j}^{1}+\cdots+(-1)^{r} b_{i j}^{r}\right) \\
& =\sum_{j} y_{j} \nabla_{x}\left(S_{r}-S_{r-1} y_{j}+\cdots+(-1)^{r} y_{j}^{r}\right)
\end{aligned}
$$

where $y_{1}, \cdots, y_{n}$ are the eigenvalues of the symmetric matrix $\left(b_{i j}\right)$. We can write $Q_{r}=\sum_{j=1}^{n} \sum_{t=0}^{r}(-1)^{r-t} y_{j} Q_{r t j}$, where $Q_{r t j}=\nabla_{x}\left(S_{t} y_{j}^{r-t}\right)$. Notice that $Q_{r t j}$ is the sum of terms of the form $\nabla_{x}\left(y_{i_{1}} y_{i_{2}} \cdots y_{i_{t}} y_{j}^{r-t}\right)$, there being exactly one such term for each choice of integers $i_{1}, i_{2}, \cdots, i_{t}$ satisfying $1 \leq i_{1}<i_{2}<\ldots$ $<i_{t} \leq n$. These terms can be classified as either Type $A$, if $j \in\left\{i_{1}, \cdots, i_{t}\right\}$, or Type $B$, if $j \notin\left\{i_{1}, \cdots, i_{t}\right\}$. Then we write $Q_{r t j}=Q_{r t j A}+Q_{r t j B}$, where $Q_{r t j A}$ $\left(Q_{r t j B}\right)$ is the sum of the terms of type $A$ (of type $B$ ) in $Q_{r t j}$. By inspection we see that $(-1)^{r-t} Q_{r t j A}+(-1)^{r-t-1} Q_{r(t-1) j B}=0$, so $Q_{r}=\sum_{j} Q_{r r j B} y_{j}$. That is, $Q_{r}$ is the sum of all the terms of the form $y_{j} \nabla_{x}\left(y_{i_{1}} y_{i_{2}} \cdots y_{i_{r}}\right), 1 \leq i_{1}<i_{2}$ $<\cdots<i_{r} \leq n, 1 \leq j \leq n, j \notin\left\{i_{1}, \cdots, i_{r}\right\}$. Thus, every summand of the form $y_{i_{1}} y_{i_{2}} \cdots \nabla_{x}\left(y_{i_{k}}\right) \cdots y_{i_{r+1}}, 1 \leq i_{1}<i_{2}<\cdots<i_{r+1} \leq n$, appearing in the expression for $\nabla_{x} S_{r+1}$ appear exactly $r$ times as a summand of $Q_{r}$. Thus $Q_{r}=$ $r \nabla_{x} S_{r+1}$.

b) Let us set $c_{i j}=t b_{i j}$ where $t$ is a new real variable, and for each $k$ let $T_{k}$ be the $k$-th elementary symmetric function of the eigenvalues of $\left(c_{i j}\right)$. Then by applying a) to the matrix $\left(c_{i j}\right)$, with $\nabla_{x}=d / d t$, and using the fact $T_{k}=$ $t^{k} S_{k}$, we see that each side of equation b) is equal to $\left(1 / t^{r}\right)(d / d t)\left(T_{r+1}\right)$.

Remark. Part b) of Lemma A is the classical Newton's formula [11].

Proof of Lemma B. The proof is by induction on $r$. The truth of the lemma in the case $r=0$ follows directly from the Codazzi equations for hypersurfaces in a space of constant curvature. Now suppose that we have proved Lemma B for $r=0, \cdots, k$. Then at the given point and for each $i$ we have

$$
\nabla_{e_{i}} S_{k+1}=\sum_{j} \nabla_{e_{j}}\left(S_{k} b_{i j}-S_{k-1} b_{i j}^{2}+\cdots+(-1)^{k} b_{i j}^{k+1}\right) .
$$

For fixed $h, 1 \leq h \leq n$, multiplying both sides of the equation by $b_{i h}$ and suming over $i=1, \cdots, n$ we get:

(*) $\quad \sum_{i} b_{i h} \nabla_{e_{i}} S_{k+1}=\sum_{i, j} b_{i h} \nabla_{e_{j}}\left(S_{k} b_{i j}-S_{k-1} b_{i j}^{2}+\cdots+(-1)^{k} b_{i j}^{k+1}\right)$.

However,

$$
\begin{aligned}
\sum_{i} b_{i h} \nabla_{e_{i}} S_{k+1} & =\sum_{i} \nabla_{e_{i}}\left(S_{k+1} b_{i h}\right)-S_{k+1} \cdot \sum_{i} \nabla_{e_{i}} b_{i h} \\
& =\sum_{i} \nabla_{e_{j}}\left(S_{k+1} b_{j h}\right)-S_{k+1} \sum_{i, j} \nabla_{e_{h}}\left(\delta_{i j} b_{i j}\right)
\end{aligned}
$$

the last equation being a consequence of the Codazzi equation. Also, 


$$
\begin{aligned}
\sum_{i, j} b_{i h} \nabla_{e_{j}}\left(S_{k} b_{i j}^{1}-S_{k-1} b_{i j}^{2}+\cdots+(-1)^{k} b_{i j}^{k+1}\right) \\
=\sum_{j} \nabla_{e_{j}}\left(S_{k} b_{h j}^{2}-S_{k-1} b_{h j}^{3}+\cdots+(-1)^{k} b_{h j}^{k+2}\right) \\
\quad-\sum_{i, j}\left(S_{k} b_{i j}^{1}-S_{k-1} b_{i j}^{2}+\cdots+(-1)^{k} b_{i j}^{k+1}\right) \nabla_{e_{h}} b_{i j},
\end{aligned}
$$

again using the Codazzi equations. Combining these equations with equation (*) we get:

$$
\begin{aligned}
\sum_{j} V_{e_{j}}\left(S_{k+1} b_{h j}-S_{k} b_{h j}^{2}+\cdots+(-1)^{k+1} b_{h j}^{k+2}\right) \\
\stackrel{(1)}{=} \sum_{i, j}\left(\left(S_{k+1} \delta_{i j}-S_{k} b_{i j}^{1}+\cdots+(-1)^{k+1} b_{i j}^{k+1}\right) \nabla_{e_{h}} b_{i j}\right) \\
=\nabla_{e_{h}}\left(\sum_{i, j}\left(S_{k+1} \delta_{i j}-S_{k} b_{i j}^{1}+\cdots+(-1)^{k+1} b_{i j}^{k+1}\right) b_{i j}\right) \\
\quad-\sum_{i, j} b_{i j} \nabla_{e_{h}}\left(S_{k+1} \delta_{i j}-S_{k} b_{i j}^{1}+\cdots\right) \\
\quad \stackrel{(2)}{=}(k+2) \nabla_{e_{h}} S_{k+2}-(k+1) \nabla_{e_{h}} S_{k+2}=\nabla_{e_{h}} S_{k+2} .
\end{aligned}
$$

The equality (1) follows from the induction hypothesis while the equality (2) follows from using b) and a) of Lemma A on the first and second terms, respectively, of the left hand side of equality (2). This completes the induction and the proof.

Proof of Theorem $A$. Let $T$ be the tensor of type $(1,1)$ on $M$ whose matrix relative to a local frame field $e_{1}, \cdots, e_{n}$ is $\left(t_{i j}\right)=\left(S_{r} \delta_{i j}-S_{r-1} b_{i j}^{1}+\cdots+\right.$ $\left.(-1)^{r} b_{i j}^{r}\right)$. Let $W^{\prime}$ be the tangent vector field $\sum_{j}\left(A, e_{j}\right) e_{j}$. Then i) $\operatorname{div}(T)=0$, for this is the content of Lemma $\mathrm{B}$. ii) If $e_{1}, \cdots, e_{n}$ are geodesic at a given point, then $\frac{1}{2}\left(W_{i, j}^{\prime}+W_{j, i}^{\prime}\right)=(A, N) b_{i j}-(A, X) \delta_{i j}$ at the point; this is a simple computation. Thus if we set $W=T\left(W^{\prime}\right)$, then by the remarks in $\S 1$ we have

$$
\begin{aligned}
& \operatorname{div}(W)=\sum_{i, j} \frac{1}{2} t_{i j}\left(W_{i, j}^{\prime}+W_{j, i}^{\prime}\right) \\
& =\sum_{j}(A, N)\left(S_{r} b_{j j}^{1}-S_{r-1} b_{j j}^{2}+\cdots+(-1)^{r} b_{j j}^{r+1}\right) \\
& \quad-\sum_{i, j}(A, X)\left(S_{r} \delta_{i j}-S_{r-1} b_{i j}+\cdots+(-1)^{r} b_{i j}^{r}\right) \delta_{i j} \\
& =U(r+1) S_{r+1}-V(n-r) S_{r} .
\end{aligned}
$$

If we express $S_{r}, S_{r+1}$ in terms of $\sigma_{r}, \sigma_{r+1}$, we get $\operatorname{div}(W)=n ! /((n-r-1) ! r !)$ $\cdot\left(U \sigma_{r+1}-V \sigma_{r}\right)$. An application of Stokes' theorem thus gives the required integral formula.

Remark. Theorem A was also proved by Katsurada in [8], where a more general result was obtained. We get this result by replacing the vector field $W^{\prime}=\sum_{i}\left(A, e_{i}\right) e_{i}$ in our proof of Theorem $\mathrm{A}$ by an arbitrary vector field 
tangent to $M$ and replacing $S^{n+1}$ by an arbitrary space $M^{\prime}$ of constant sectional curvature. If $M^{\prime}$ is flat, we get the Hsiung formulas [7]. However Katsurada does not explicitly consider the situation in Theorem A.

Corollary A. Suppose that for some $r, 1 \leq r \leq n-1$, either

i) $\sigma_{r}$ and $\sigma_{r-1}$ are constant on $M, U>0$, and at some point of $M$ all the principal curvatures are non-zero, or

ii) $\sigma_{r}$ and $\sigma_{r+1}$ are constant and $V>0$.

Then $X$ embeds $M$ as a hypersphere in $S^{n+1}$.

Proof. i) By Theorem $A, \int_{M}\left(\sigma_{r} U-\sigma_{r-1} V\right) d V=0=\int_{M}\left(\sigma_{r+1} U-\sigma_{r} V\right) d V$. If we multiply the left hand side by the constant $\sigma_{r}$ and the right hand side by the constant $\sigma_{r-1}$ and then subtract the resulting equations we get $\int_{M} U\left(\sigma_{r}^{2}-\right.$ $\left.\sigma_{r-1} \sigma_{r+1}\right) d V=0$. But Newton's inequality [6] says that $\sigma_{r}^{2}-\sigma_{r-1} \sigma_{r+1} \geq 0$ and by hypothesis $U>0$, so we must have $\sigma_{r}^{2}-\sigma_{r-1} \sigma_{r+1} \equiv 0$. Now if $q$ is a point of $M$ at which no principal curvature vanishes, then so is any point close to $q$. Also since the Newton inequality is in this case the equation $\sigma_{r}^{2}-\sigma_{r-1} \sigma_{r+1}=0$, then every such $q$ is an umbilic of $M$ (c.f. [6]). However the principal curvatures are all constant in any open connected set of umbilics. Thus the subset of $M$ consisting of all umbilics at which the principal curvature equals that at $q$, clearly a closed subset, is also a nonempty open subset and thus equals the connected set $M$. Hence $M$ is a hypersphere.

ii) By similar reasoning we conclude from the constancy of $\sigma_{r}$ and $\sigma_{r+1}$ that $\int_{M} V\left(\sigma_{r}^{2}-\sigma_{r+1} \sigma_{r-1}\right) d V \equiv 0$, and since $V>0$ we conclude that $\sigma_{r}^{2}-\sigma_{r+1} \sigma_{r-1}$ $\equiv 0$. It can be shown, by considering the place where $V$ is minimum, that there exists a point of $M$ at which all the principal curvatures are positive. With this fact we proceed as in i) to finish the proof,

Corollary B. Suppose that for some $r, 1 \leq r \leq n-1, \sigma_{r}=0, \sigma_{r+1}$ has constant sign and $U>0$. Then $\sigma_{r+1}=0$.

Proof. If $\sigma_{r}=0$, then by Theorem A, $\int_{M} U \sigma_{r+1} d V=0$. But $U>0$ and $\sigma_{r+1}$ has constant sign, thus $\sigma_{r+1}=0$.

Remark. The case $r=1$ of Corollary B was considered by De Giorgi [5] and Simons [9]. It is a special case of Theorem $\mathrm{C}$ in $\S 2$.

\section{A generalization to higher codimensions}

A major advantage of our proof of Theorem A over that of Katsurada is that with our formalism the theorem is easier to generalize, which we do in this section.

Notation. We consider an immersion $X: M \rightarrow S^{n+p}$. Our local calcula- 
tions are done relative to an adapted positively oriented orthonormal frame field $e_{1}, \cdots, e_{n+p}$ on $S^{n+p}$, i.e., $e_{1} \cdots e_{n}$ is tangent to $X(M)$ and positively oriented to $M$. We follow the index conventions $1 \leq i, j, k \leq n$ and $n+1 \leq$ $r, s \leq n+p$. The matrix of the vector-valued second fundamental form relative to $e_{1} \cdots e_{n+p}$ is $b_{r i j}$, the mean curvature vector is $\sigma_{1}=(1 / n) \sum_{r} \sum_{j} b_{r j j} e_{r}$ and the second mean curvature is $\sigma_{2}=(1 / n(n-1)) \sum_{r} \sum_{i, j}\left(b_{r i i} b_{r j j}-b_{r i j}^{2}\right)$. The exterior algebra of $R^{n+p+1}$ is denoted by $\Lambda$, and the subspace spanned by $m$ planes by $\Lambda^{m}$. We define non-negative functions $B_{r}, B$ on $M$ by $B_{r}^{2}=\sum_{i, j} b_{r i j}^{2}$ and $B^{2}=\sum_{r} B_{r}^{2}$. Note that $B^{2}=n^{2}\left|\sigma_{1}\right|^{2}-n(n-1) \sigma_{2}$, and the vanishing of $B^{2}$ on $\mathrm{M}$ is equivalent to the immersion being totally geodesic. We set $N=e_{n+1}$ $\wedge \cdots \wedge e_{n+p} \in \Lambda^{p}$. Thus $N$ is the (smooth) field of oriented unit normal $p$ planes of $M$ in $S^{n+p}$. $N$ is independent of the choice of local frame field and thus is globally defined on $M$. For each $r$ we set $X_{r}=e_{n+1} \wedge \cdots \wedge e_{r-1} \wedge$ $X \wedge e_{r+1} \wedge \cdots \wedge e_{n+p}$. Let $A_{n+1}, A_{n+2}, \cdots, A_{n+p}$ be an orthonormal set of vectors in $E^{n+p+1}$, and set $A=A_{n+1} \wedge \cdots \wedge A_{n+p} \in \Lambda^{p}$. In terms of the standard inner product on $\Lambda^{p}$ (cf. [10]) set $U=(N, A), V_{r}=\left(X_{r}, A\right)$ and

$$
h_{r s i j}=\left(e_{n+1} \wedge \cdots \wedge e_{r-1} \wedge e_{i} \wedge e_{r+1} \wedge \cdots \wedge e_{s-1} \wedge e_{j} \wedge e_{s+1} \wedge \cdots \wedge e_{n+p}, A\right),
$$

if $r \neq s$ and $h_{r r i j}=0$. Note that

i) $h_{r s i j}=-h_{s r i j}=-h_{r s j i}$,

ii) $U^{2}+\sum_{r<s} \sum_{i<j} h_{r s i j}^{2} \leq 1$;

for $U$ and $h_{r s i j}$ are just some of the components of the unit $p$-plane $A$ relative to the orthonormal basis of $\Lambda^{p}$ generated by the vectors $e_{i}, e_{r}, X$ in $E^{n+p+1}$.

Theorem B. a) Let $A^{\prime}$ be any vector in $E^{n+p+1}$. Then

$$
\begin{gathered}
\int_{M}\left(\left(\sigma_{1}, A^{\prime}\right)-\left(X, A^{\prime}\right)\right) d V=0, \\
\text { b) } \int_{M}\left[n(n-1) \sigma_{2} U-n(n-1) \sum_{r}\left(\sigma_{1}, e_{r}\right) V_{r}\right. \\
\left.+2 \sum_{r<s} \sum_{i<j} \sum_{k}\left(b_{r i k} b_{s j k}-b_{r j k} b_{s i k}\right) h_{r s i j}\right] d V=0 .
\end{gathered}
$$

Proof. a) Consider the real-valued function $z=\left(X, A^{\prime}\right)$. One easily computes that $\Delta z=n\left(\sigma_{1}, A^{\prime}\right)-n\left(X, A^{\prime}\right)$. Hence an application of Stokes' theorem gives the required integral formula.

b) Define a vector field $W$ by the formula $W=\sum_{j} W_{j} e_{j}$, where

$$
\begin{aligned}
W_{j}=\sum_{i} \sum_{r} & \left(n\left(\sigma_{1}, e_{r}\right) \delta_{i j}-b_{r i j}\right) \\
& \cdot\left(e_{n+1} \wedge \cdots \wedge e_{r-1} \wedge e_{i} \wedge e_{r+1} \wedge \cdots \wedge e_{n+p}, A\right) .
\end{aligned}
$$


Clearly $W$ does not depend on the choice of orthonormal frame field. Then b) follows by computing $\operatorname{div}(W)$ and applying Stokes' theorem. The computation of $\operatorname{div}(W)$ uses the Codazzi equations, the symmetry of $b_{r i j}$ in $i$ and $j$ and the antisymmetries i) of $h_{r s i j}$. q.e.d.

Despite the relative complexity of the equation b) in Theorem B we are able to draw from it an interesting geometric conclusion. To do this we need a simple algebraic lemma, whose proof can be found in [2].

Lemma C. Suppose that $C=\left(c_{i j}\right)$ and $D=\left(d_{i j}\right)$ are symmetric $n \times n$ matrices. Then

$$
\sum_{i, k}\left(\sum_{j}\left(c_{i j} d_{j k}-c_{k j} d_{j i}\right)\right)^{2} \leq 2\left(\sum_{i, j} c_{i j}^{2}\right)\left(\sum_{k, l} d_{k l}^{2}\right) .
$$

We use this lemma to estimate the quantity

$$
Q=\sum_{r<s} \sum_{i<k} \sum_{j}\left(b_{r i j} b_{s j k}-b_{s i j} b_{r j k}\right) h_{r s i k}
$$

in terms of $B^{2}$. First of all, the Cauchy-Schwartz inequality implies that

$$
Q^{2} \leq\left(\sum_{r<s} \sum_{i<k}\left(\sum_{j}\left(b_{r i j} b_{s j k}-b_{r k j} b_{s i j}\right)\right)^{2}\right)\left(\sum_{r<s} \sum_{i<k} h_{r s i k}^{2}\right) .
$$

By ii), $\sum_{r<s} \sum_{i<k} h_{r s i k}^{2} \leq 1-U^{2}$. By Lemma C,

$$
\begin{aligned}
& \sum_{i<k}\left(\sum_{j}\left(b_{r i j} b_{s j k}-b_{r k j} b_{s i j}\right)\right)^{2} \\
& \quad=\frac{1}{2} \sum_{i, k}\left(\sum_{j}\left(b_{r i j} b_{s j k}-b_{r k j} b_{s i j}\right)\right)^{2} \leq\left(\sum_{i, j} b_{r i j}^{2}\right)\left(\sum_{i, j} b_{s i j}^{2}\right) \\
& \quad=B_{r}^{2} B_{s}^{2} \quad(\text { for fixed } r, s) .
\end{aligned}
$$

Thus

$$
\sum_{r<s} \sum_{i<k}\left(\sum_{j}\left(b_{r i j} b_{s j k}-b_{r k j} b_{s i j}\right)\right)^{2} \leq \sum_{r<s} B_{r}^{2} B_{s}^{2} .
$$

Now the Newton inequalities imply that

$$
\sum_{r<s} B_{r}^{2} B_{s}^{2} \leq(p(p-1) / 2)\left(\sum_{r} B_{r}^{2} / p\right)^{2}=((p-1) / 2 p) B^{4} .
$$

Combining all these inequalities we see that

$$
Q \leqq \sqrt{(p-1) / 2 p} \cdot \sqrt{1-U^{2}} B^{2} .
$$

Theorem C (DeGiorgi-Simons). Suppose that $X: M \rightarrow S^{n+p}$ is a minimal immersion of the compact oriented n-manifold $M$ into $S^{n+p}$ such that function $U$ defined above satisfies the hypothesis $U>\sqrt{(2 p-2) /(3 p-2)}$. Then $(M, X)$ is totally geodesic. In particular, this is the case if $U>\sqrt{2 / 3}$, inde- 
pendently of $p$.

Proof. Theorem B and the estimate for $Q$ imply that if $\sigma_{1}=0$ then

$$
0=\int_{M}\left(-B^{2} U+2 Q\right) d V \leq \int_{M}\left(-B^{2} U+2 \sqrt{(p-1) / 2 p} \sqrt{1-U^{2}} B^{2}\right) d V .
$$

If $U>\sqrt{(2 p-2) /(3 p-2)}$, then we easily see that

$$
2 \sqrt{\frac{p-1}{2 p}} \sqrt{1-U^{2}}-U<2 \sqrt{\frac{p-1}{2 p}} \cdot \sqrt{\frac{p}{3 p-2}}-\sqrt{\frac{2 p-2}{3 p-2}}=0 .
$$

This, together with the integral inequality at the beginning of this proof, implies that $B^{2} \equiv 0$ on $M$, i.e., $(M, X)$ is totally geodesic.

Remark. Theorem $C$ improves Theorem 5.2 of [9], since the hypothesis there is $U>\sqrt{(p+1) /(p+2)}$. Thus our result is better in every codimension, and more importantly gives the single bound $\sqrt{2 / 3}$ which will work for any codimension. For further discussion, see the appendix.

\section{Appendix}

In this appendix we consider the DeGiorgi-Simons theorem (Theorem C) in a different light. We use the notation of that theorem.

In [9] Simons asks if we can weaken the hypothesis on $U$ in Theorem $\mathrm{C}$ to $U>0$. Unfortunately we are unable to answer this question, but we can derive a weaker form (Theorem D) of the DeGiorgi-Simons theorem which shows more clearly why one has to assume that $U$ is fairly far from 0 .

First of all let us indicate the geometric significance of the nonvanishing of $U$. Consider the map $Y=f \cdot\left(X-\sum_{s}\left(X, A_{s}\right) A_{s}\right): M \rightarrow S^{n}$, where $S^{n}$ is the great $n$-sphere in $S^{n+p}$ perpendicular to $A_{n+1}, \cdots, A_{n+p}$, and $f=$ $1 / \sqrt{1-\sum_{s}\left(X, A_{s}\right)^{2}}$. If $M$ is compact and connected, then one easily checks that $Y$ is a diffeomorphism if and only if $U \neq 0$ on $M$.

Now let us consider the cone over the immersion $(M, X)$. This is the immersion $\left(M^{\prime}, X^{\prime}\right)$, where $M^{\prime}=M \times(0, \infty)$, so $M^{\prime}$ is of dimension $n+1$, and $X^{\prime}: M^{\prime} \rightarrow E^{n+p+1}$ is defined as $X^{\prime}(q, t)=X(q) \cdot t, q \in M, t \in(0, \infty)$. It is wellknown (cf. [9]) that the immersion $(M, X)$ into $S^{n+p}$ is minimal if and only if $\left(M^{\prime}, X^{\prime}\right)$ is a minimal immersion into $E^{n+p+1}$. Also the unit normal $p$-plane on $M^{\prime}$ at $(q, t)$ is independent of $t$. The inner product $U^{\prime}(q, t)$ of this normal $p$ plane and the fixed $p$-plane $A_{n+1} \wedge A_{n+2} \wedge \cdots \wedge A_{n_{+} p}$ is still $U(q)>0$.

From now on our index conventions are $0 \leq i, j, k, l \leq n, n+1 \leq r, s \leq$ $n+p, n \geq 2$. Let $A_{0}, \cdots, A_{n}$ be vectors in $E^{n+p+1}$ such that $A_{0}, \cdots, A_{n}$, $A_{n+1}, \cdots, A_{n+p}$ form an orthonormal basis for $E^{n+p+1}$. Let $E^{n+1}$ be the vector subspace spanned by $A_{0}, \cdots, A_{n}$. Set $x_{j}=\left(A_{j}, X^{\prime}\right)$ and $y_{r}=\left(A_{r}, X^{\prime}\right)$. Then 
the functions $\left(x_{0}, x_{1}, \cdots, x_{n}\right)$ define a diffeomorphism $Y^{\prime}$ between $M^{\prime}$ and the open set $E^{n+1} \sim\{0\}$ in $E^{n+1}$ and in particular the $x_{j}$ form a global coordinate system on $M^{\prime}$. This is true, because $f \cdot Y^{\prime}$ is the cone over the diffeomorphism $Y: M \rightarrow S^{n}$ and $E^{n+1} \sim\{0\}$ is the cone over $S^{n}$.

Since the $x_{j}$ are global coordinates for $M^{\prime}$ we shall view the functions $y_{r}$ as functions of the $x_{j}$. Thus $X^{\prime}(M)$ has the nonparametric representation as the set of all points $\left(x_{0}, \cdots, x_{n}, y_{n+1}\left(x_{0}, \cdots x_{n}\right), \cdots, y_{n+p}\left(x_{0}, \cdots, x_{n}\right)\right)$ with $\left(x_{0}, \cdots, x_{n}\right) \neq(0, \cdots, 0)$.

The metric tensor of $\left(M^{\prime}, X^{\prime}\right)$ relative to the coordinates $x_{0}, \cdots, x_{n}$ is

$$
g_{i j}=\left(\frac{\partial X^{\prime}}{\partial x_{i}}, \frac{\partial X^{\prime}}{\partial x_{j}}\right)=\delta_{i j}+\sum_{r} \frac{\partial y_{r}}{\partial x_{i}} \cdot \frac{\partial y_{r}}{\partial x_{j}} .
$$

We denote the inverse of the matrix $\left(g_{i j}\right)$ by $\left(g^{i j}\right)$, and the Christoffel symbols (of the second kind) by $\Gamma_{j k}^{i}$. If $z$ is any smooth function on $M$, then its Laplacian is given by (cf. [3])

$$
\Delta z=\sum_{i, j=0}^{n} g^{i j}\left(\frac{\partial^{2} z}{\partial x_{i} \partial x_{j}}-\sum_{k=0}^{n} \Gamma_{i j}^{k} \frac{\partial z}{\partial x_{k}}\right) .
$$

Also a necessary and sufficient condition for an immersion into Euclidean space to be minimal is that the coordinate functions be harmonic [9].

Thus in the case of the minimal cone $\left(M^{\prime}, X^{\prime}\right)$ we must have $\Delta x_{j}=\Delta y_{r}=$ 0 . However,

$$
\Delta x_{l}=\sum_{i, j=0}^{n} g^{i j}\left(\frac{\partial^{2} x_{l}}{\partial x_{i} \partial x_{j}}-\sum_{k=0}^{n} \Gamma_{i j}^{k} \frac{\partial x_{l}}{\partial x_{k}}\right)=-\sum_{i, j=0}^{n} g^{i j} \Gamma^{l}{ }_{i j},
$$

so the condition $\Delta y_{r}=0$ becomes

$$
\sum_{i, j=0}^{n} g^{i j} \frac{\partial^{2} y_{r}}{\partial x_{i} \partial x_{j}}=0
$$

That is, each $y_{r}$ satisfies the second order elliptic partial differential equation $(*)$. The $y_{r}$ are smooth except for a possible isolated singularity at the origin. Since the $y_{r}$ are conical functions, i.e., $y_{r}(t q)=t y_{r}(q), t$ real, we know that if the origin is not a singular point of the $y_{r}$, then they must be linear functions of the $x_{j}$. In this case $X(M)$ is a clearly great $n$-sphere in $S^{n+p}$.

In order to show that if $U$ is sufficiently close to 1 on $M$ then the $y_{r}$ are smooth at 0 , we shall need a result from the theory of elliptic partial differential equations (cf. [4, p. 132]).

Proposition B. Suppose that $z=z\left(x_{0}, \cdots, x_{n}\right)$ is a smooth solution of $a$ uniformly elliptic second order partial differential equation $\sum_{i, j=0}^{n} a_{i j} \frac{\partial^{2} z}{\partial x_{i} \partial x_{j}}=0$, $n \geq 2$, with an isolated singularity at the origin. If $z$ is $\mathcal{O}\left(r^{1-n+\delta}\right)$ at the origin 
for some $\delta>0$ (in particular if $z$ is Lipschitz at the origin), and if $\lim _{x \rightarrow 0} \sup _{x \rightarrow 0}$ $\left|a_{i j}(x)-\delta_{i j}\right|$ is sufficiently small (although we don't assume the $a_{i j}$ are continuous at the origin), then $z$ is actually smooth at the origin.

To be able to apply Proposition B to our situation we first notice that each $y_{r}$, being a conical function, is Lipschitz at the origin. Secondly we notice that if $U$ is close to 1 , then $g_{i j}$, and thus $g^{i j}$, is close to $\delta_{i j}$ at each point (i.e., uniformly). Indeed it is easy to show that $1 \geq 1 / \sqrt{1+\sum_{k=0}^{n}\left(\partial y_{r} / \partial x_{k}\right)^{2}} \geq|U|$ and thus if $U$ close to 1 , then $\partial y_{r} / \partial x_{k}$ is close to 0 for each $r, k$ and therefore $g_{i j}$ is close to $\delta_{i j}$. In light of our earlier remarks, we have thus proved

Theorem D. If $M^{n}$ is a compact minimal submanifold in $S^{n+p}$ such that the function $U$ is sufficiently near 1 on $M$, then $M$ is a totally geodesic submanifold of $S^{n+p}$.

\section{Bibliography}

[1] S. S. Chern, Integral formulas for hypersurfaces in Euclidean space and their applications to uniqueness theorems, J. Math. Mech. 8 (1959) 947-956.

[2] S. S. Chern, M. do Carmo \& S. Kobayashi, Minimal submanifolds of a sphere with second fundamental form of constant length, Functional analysis and related fields, Proc. Conf. in Honor of Marshall Stone, Springer, 1970.

[ 3 ] L. P. Eisenbart, Riemannian geometry, Princeton University Press, Princeton, 1926.

[4] D. Gilbarg, Some local properties of elliptic equations, Proc. Sympos. Pure Math. Vol. IV, Amer. Math. Soc., 1961, 127-141.

[5] E. De Giorgi, Una estensione del teorema di Bernstein, Ann. Scuola Norm. Sup. Pisa 19 (1965) 79-85.

[6] G. H. Hardy, J. E. Littlewood \& G. Polya, Ineqalities, Cambridge University Press, Cambridge, 1934.

[7] C. C. Hsiung, Some integral formulas for closed hypersurfaces, Math. Scand. 2 (1954) 286-294.

[8] Y. Katsurada, Generalized Minkowski formulas for closed hypersurfaces in Riemann space, Ann. Mat. Pura Appl. 57 (1962) 283-293.

[9] J. Simons, Minimal varieties in riemannian manifolds, Annals of Math. 88 (1968) $62-105$.

[10] S. Sternberg, Lectures on differential geometry, Prentice-Hall, Englewood Cliffs, N. J, 1964.

[11] B. L. van der Waerden, Modern algebra, Vol. I, Frederick Unger, New York, 1949. 
